\title{
Enteral Migration of Feeding Jejunostomy tube: Report of a Case
}

\author{
Authors \\ Deshraj Chawla ${ }^{1}$, Mahesh Kumar Sonwal ${ }^{2}$, Harsh Kumar ${ }^{3}$, Neeraj Kumar Dewanda ${ }^{4}$, \\ Shivcharan Bairwa ${ }^{5}$ Ritu Kumari $^{6}$, Pankaj Jhang ${ }^{7}$ \\ ${ }^{1,3}$ Assistant Professor, Department General Surgery Government Medical College, Kota \\ ${ }^{2}$ Senior Resident, Department General Surgery Government Medical College, Kota \\ ${ }^{4}$ Proffesor and Unit Head, Department General Surgery, Government Medical College, Kota \\ ${ }_{5,6,7}$ Resident, Department General Surgery, Government Medical College, Kota \\ Corresponding Author \\ Mahesh Kumar Sonwal \\ Senior Resident, Department General Surgery Government Medical College, Kota \\ Email: sonwalsms@gmail.com, Contact No: 9785332419
}

\section{Abstract}

We report a rare complication of the migration of a feeding jejunostomy tube inside the gut. A Stamm type jejunostomy was performed in a patient with an unresectable esophageal cancer using a 16-F ryles tube, at another center. Two months later, she presented because of the "disappearance" of the tube. A clinical examination revealed a mature jejunostomy tract, skin erosion at the site where the suture was placed, and absence of the tube. There were no signs of intestinal obstruction or peritonitis. Plain abdominal X-ray examination showed the tube inside the abdomen.

The patient was treated initially conservatively with serial radiographs which showed no migration of tube for 4 days. Therefore, an exploratory laprotomy was done with removal of old tube with new feeding jejunostomy at previous entrostomy site.

An awareness of this complication and its appropriate treatment is important given the widespread use of feeding jejunostomy tube.

Keywords: Ryles tube, Feeding jejunostomy, Enteral nutrition, Complications, Migration.

\section{Introduction}

Feeding jejunostomy is a method that is widely used for enteral nutrition in patients with normally functioning intestines but unable to take orally. Several different surgical techniques and tube type are presently used, and despite its technical simplicity, serious complications associated with the feeding tube have been reported. We report a rare complication consisting of an enteral migration of feeding jejunostomy tube.

\section{Case Report}

A 30-year-old female with an unresectable esophageal cancer had a surgical Stamm jejunostomy performed for enteral nutrition at another center.

Two months later, she presented to our emergency department because of the "disappearance" of the tube. A clinical examination revealed a mature jejunostomy tract, skin erosion at the site where the suture was placed, and no sign of the tube. 
There were no signs of infection, leakage, obstruction, volvulus or perforation. A plain abdominal X-ray examination revealed the Ryles tube to be inside the abdomen. Other routine investigations were within normal limit. Patient was initially treated conservatively, with serial plain abdominal x-ray, but there was no further migration of tube for 4 days, therefore an exploratory laprotomy was planned.

On exploration, the feeding jejunostomy tube (Ryles tube) was found inside the distal ileum and ascending colon. The Ryles tube was removed by retrograde milking from the old jejunal entrostomy site. A new ryles tube was inserted as feeding jejunostomy with fixation of Ryles tube by using silk 2-0 RB suture at old entrotomy site and fix to parietal peritoneum by using silk 2-0 RB.

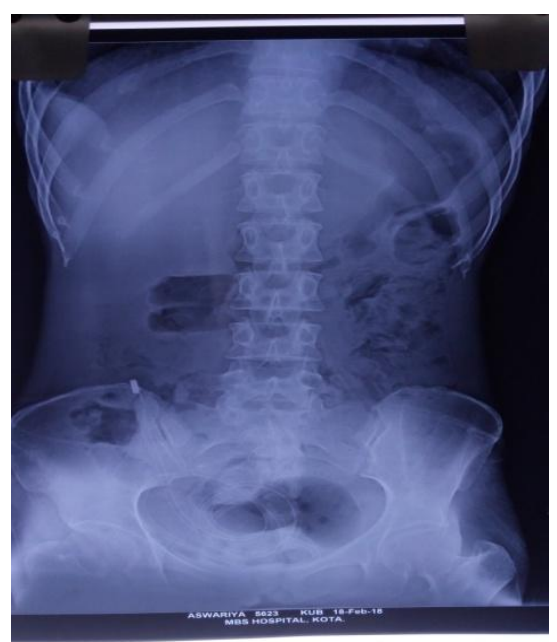

Fig 1 Plain radiograph showing intra abdominal position of migrated FJ Tube

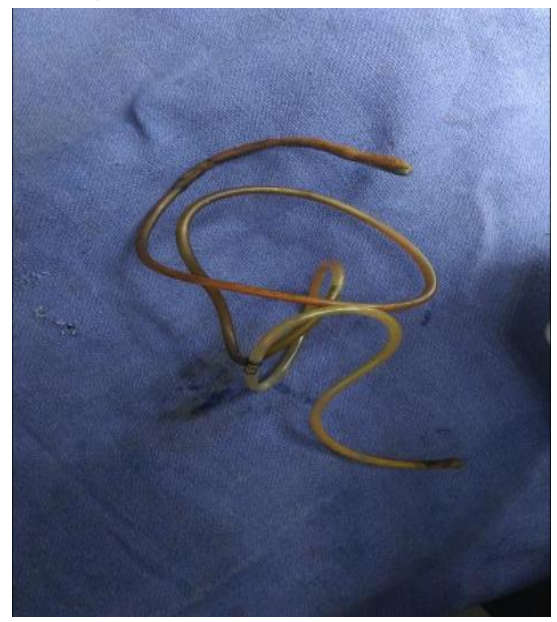

Fig 2 Migrated feeding jejunostomy tube after removal

\section{Discussion}

Jejunostomy tube feeding is widely used for the nutritional support of patients who are unable to take orally but have normally functioning intestines. Despite its benefits compared to parenteral nutrition, enteral tube feeding is not without risk. Apart from metabolic disturbances, 1various complications associated with the migration of the feeding tubes are known to occur, 2 and it can sometimes results in small bowel obstruction, 3,4 intussusception, 5 and obstructive jaundice. 6 Gowen7 reported nine occurrences of distal migration and 19 episodes of balloon rupture in four patients who had a Foley catheter feeding gastrostomy, and they recommended the use of a mushroom tipped catheter to avoid these complications. Secure fixing either by direct suturing of the catheter to the skin or by using an external retention ring on the catheter has also been proposed for the prevention of tube migration. 2

In this case, a ryles tube No $16 \mathrm{~F}$ was used for the construction of a surgical jejunostomy, and the tube was securely fixed to the skin. However, skin erosion allowed the tube to free itself, and it subsequently migrated distally due to intestinal peristalsis. In a previous report, a Foley's catheter gastrostomy tube migrated into the small bowel resulting in an obstruction, which was relieved by percutaneous balloon aspiration that allowed the tube to migrate into the colon. 8 Eventually the tube was impacted into the sigmoid colon where it was reached by a colonoscopy and then was retrieved with a snare. In two other cases, the distal migration of the jejunostomy tube was associated with retrograde plication of the small bowel over the tube, thus bypassing most of the jejunum and resulting in severe diarrhea, hyper peristalsis, and malnutrition.9,10 In both cases the condition was discovered at autopsy. In the only other similar reported case, a jejunostomy with a Foley catheter migrated through the entire intestine and passed in the feces without the need for a surgical exploration. 
However, any kind of long-standing feeding tube that is placed either surgically or endoscopically can migrate, and as the placement of such tubes becomes more common, an awareness of this complication and its successful management has thus become increasingly important. Family members, nursing home personnel, or other health care providers who care for patients with jejunostomies for long-term enteral nutrition, must be instructed in detecting a loosening of tube fixation or tube displacement among other complications.

\section{Conclusion}

When enteral migration of a jejunostomy feeding tube occurs, the patient can be treated conservatively provided that there are no signs of an acute abdomen. Serial abdominal radiographs should be taken to follow the advancement of the tube within the intestinal lumen. If the tube in not spontaneously eliminated, as seen in our patient, and can be reached endoscopically, then a colonoscopic retrieval should be attempted. Surgical removal should be a last treatment option. The migrated tube may removed by retrograde milking from the previous entrostomy site, thus avoiding a new entostomy. The external portion of the tube can be marked with a marker or a tape, and instructions should be given to keep the marked point at the skin level. ${ }^{8}$

\section{References}

1. Yagi M, Hashimoto T, Nezuka H, Ito $H$, Tani T, Shimizu K, et al. Complications associated with enteral nutrition using catheter jejunostomy after esophagectomy. Surg Today 1999;29:214-8.

2. Tapia J, Murguia R, Garcia G, de los Monteros PE, Onate E. Jejunostomy: techniques, indications, and complications. World J Surg 1999;23:596-602.

3. Haws EB, Sieber WK, Kiesewetter WB. Complications of tube gastrostomy in infants and children. 15-year review associated of 240 cases. Ann Surg 1966;164:284-90.

4. O'Dell KB, Gordon RS, Becker LB. Gastrostomy tube transmigration: a rare cause of small bowel obstruction. Ann Emerg Med 1991;20:817-9.

5. Goldman DE, Grier JF. The migrating gastrostomy tube. Gastrointest Endosc 1995;42:102-3.

6. Konda J, Ruggle P. Prolapse of Foley catheter gastrostomy tube causing obstructive jaundice. Am J Gastroenterol 1981;76:353-5.

7. Gowen GF. The management of complications of Foley feeding gastrostomies. Am Surg 1988;54:582-5.

8. Pereira MC, Mersich K. Foley catheter gastrostomy tube migration: small bowel obstruction relieved by percutaneous balloon aspiration. Gastrointest Endosc 1991;37:372-4.

9. Prahlow JA, Barnard JJ. Jejunostomy tube failure: malnutrition caused by intraluminal antegrade jejunostomy tube migration. Arch Phys Med Rehabil 1998;79:453-5.

10. Opeskin K, Lee KA. Failure of a feeding jejunostomy. Med Sci Law 1993;33:2636.

11. Jain BK, Mehta RB. Enteral migration of a Foley catheter after jejunostomy. Can J Surg 1991;34:106-7.

12. Kadakia SC, Cassady M, Shaffer RT. Comparison of Foley catheter as a replacement gastrostomy tube with commercial replacement gastrostomy tube: a prospective randomised study. Gastrointest Endosc 1994;40:188-93. 